\title{
Ocular findings in sleep apnoea patients using continuous positive airway pressure
}

A Kadyan', J Asghar², L Dowson ${ }^{3}$ and

S Sandramouli²

\begin{abstract}
Purpose To describe ocular findings in patients with established obstructive sleep apnoea hypopnoea syndrome (OSAHS) using continuous positive airway pressure (CPAP). Methods One hundred and fifteen referrals investigated for OSAHS were included. Patients with OSAHS were compared with those with normal sleep study controls. Subgroup analysis for CPAP users and nonusers was also carried out.
\end{abstract}

Results OSAHS patients $(n=89)$ compared with the controls $(n=26)$ had higher ocular irritation symptoms $(P<0.001)$, abnormal tear break-up time $(P<0.05)$ with increased upper $(P<0.001)$ and lower $(P<0.001)$ lid laxity. Floppy eyelid syndrome (FES) was noted in $31.5 \%(28 / 89)$ OSAHS patients vs $3.8 \%(1 / 26)$ controls $(P=0.005)$. Open angle glaucoma prevalence in OSAHS patients $(3 / 89,3.4 \%)$ was similar to the controls $(1 / 26,3.8 \%)(P=0.92)$. Sixty-seven $(75.3 \%)$ OSAHS patients were using CPAP (average duration: $19.6 \pm 15.3$ months). All CPAP users maintained a supine sleep posture to prevent mask edge leaks. A fifth of CPAP users (14/67) had experienced earlier episodes of conjunctivitis secondary to leaks. CPAP users had similar upper and lower lid laxity $(P=0.746$ and 0.633$)$ to non-CPAP users, but a better tear film $(P=0.029)$ and less ocular irritation $(P=0.134)$.

Conclusion OSAHS patients showed increased ocular irritation, abnormal tear film, lid laxity, and FES. The prevalence of glaucoma in our series was similar to normal population data of $2 \%, P=0.429$, and may relate to use of CPAP in majority of the patients. More stable tear film in CPAP users was probably secondary to the supine sleep postures necessarily adopted with CPAP use.
Eye (2010) 24, 843-850; doi:10.1038/eye.2009.212; published online 14 August 2009

Keywords: obstructive sleep apnoea; continuous positive airway pressure; floppy eye lid syndrome; glaucoma

\section{Introduction}

Obstructive sleep apnoea hypopnoea syndrome (OSAHS) is characterised by sleep induced upper airway narrowing leading to symptomatic sleep disturbance. ${ }^{1}$ The pathophysiological consequences of years of sleep fragmentation and deprivation can affect almost every organ in the body and patients often present to non-sleep specialists with symptoms related to, caused by, or exacerbated by OSAHS. ${ }^{2}$ Recognition of these varied manifestations has passed from relative obscurity to mainstream clinical activity in several specialities including ophthalmology over the last 10-15 years. Ocular associations reported with OSAHS include floppy eyelid syndrome (FES), blepharoptosis, lower lid ectropion, blepharochalasis, eyelash ptosis, lacrimal gland prolapse, papillary conjunctivitis, filamentary or infectious keratitis, recurrent corneal erosion, keratoconus, optic neuropathy, papilloedema, and glaucoma. ${ }^{3-13}$

Specific disease management uses the basic concept of gentle pressurisation of the upper airway during sleep to prevent the collapse of the pharynx. Continuous positive airway pressure (CPAP) administered through a nasal or face mask has remained the mainstay of treatment in OSAHS since its advent in the 1980s. ${ }^{13}$ Non-specific behavioural measures such as weight loss, avoiding smoking, alcohol, and sedatives can impact on symptom control, but are inadequate for moderate to severe
${ }^{1}$ Birmingham and Midland Eye Centre, Birmingham, UK

${ }^{2}$ Wolverhampton and Midland Counties Eye Infirmary, Wolverhampton, UK

${ }^{3}$ Department of Medicine, New Cross Hospital, Wolverhampton, UK

Correspondence: A Kadyan, Birmingham and Midland Eye Centre, City Hospital, Dudley Road, Birmingham B18 7QH, UK Tel: + 447766753258 ; Fax: + 441902504050 E-mail: anjukadyan@ btinternet.com

Received: 7 May 2009 Accepted in revised form: 28 June 2009

Published online: 14 August 2009

Paper presented at Ophthalmology Showcase session, Royal College of Ophthalmologists Annual Congress, Liverpool, 2008 
disease. Effect of these treatment measures on the ocular manifestations is less well documented, as most

literature documents ocular changes at time of diagnosis of the sleep disorder. Isolated case reports in literature have suggested reversal of symptoms of FES accompanied with significant weight $\operatorname{loss}^{14}$ and initiation of CPAP.$^{15}$ More recently, effects of CPAP on intraocular pressure and performance in visual field (VF) testing have been reported. ${ }^{16,17}$ In addition, leaking masks used for CPAP treatment can cause ocular irritation and even induce conjunctivitis. ${ }^{18,19}$

In this study, we describe the ocular findings in patients with established moderate to severe OSAHS, majority of who were on treatment with CPAP.

\section{Materials and methods}

\section{Statement of ethics}

The study protocol was approved by the Local Research and Ethics Committee, convened by the Birmingham and Black Country Strategic Health Authority, UK. The study followed the tenets of the 1964 Declaration of Helsinki. Informed consent was obtained from all participants on the day of their appointment. The only exclusion criteria were age $<18$ years or adults unable to provide informed consent.

\section{Participants}

Patients evaluated for OSAHS at a tertiary referral centre in the United Kingdom over a period of 12 months (January-December 2005) were invited to have an ophthalmic examination. Two hundred and sixty-nine patients were investigated in this period: 125 were classified as having OSAHS and 144 had negative sleep studies. 89/125 (71.2\%) OSAHS patients and 26/144 $(18.05 \%)$ controls were included in our study. Ophthalmic examinations were carried out from June 2006 to February 2007. The demographic (age, sex, body mass index (BMI)) and sleep data (oxygen desaturation index, ODI) of the participants were similar to the nonresponders. Further, assuming that the patients with symptoms would be over-represented in the group who accepts a free eye examination, we included the control group of patients investigated, but found to have normal sleep studies to validate our results.

\section{Ophthalmic examination}

At the time of appointment, a complete medical and ocular history was recorded, including symptoms of ocular irritation at time of appointment (redness, tearing, dry sensation, mucous discharge, foreign body sensation, episodes of blurred vision, eye rubbing, lid swelling), spontaneous lid eversion, preferred sleeping posture, and change in earlier eye symptoms after initiation of CPAP.

Horizontal lid distraction distances (LDD) were measured based on the technique described by Liu and Stasior. ${ }^{20}$ Lid distraction $>5 \mathrm{~mm}$ for upper lids and $>6 \mathrm{~mm}$ for lower lids was considered as significant and indicative of increased laxity. Average eyelid distraction distance for upper and lower lids was calculated by averaging values from both eyes. FES as described by Culbertson and Ostler ${ }^{21}$ included the presence of lax, rubbery easily evertible lids with chronic papillary conjunctivitis. The lid laxity measurements have been shown to vary widely in earlier studies and were used primarily as an objective measure of our findings. ${ }^{5} \mathrm{We}$ based the diagnosis of FES on subjective assessment of easily evertible lids, presence of papillary conjunctivitis, and symptoms of ocular irritation similar to earlier studies. ${ }^{5,14}$

Tear film was assessed using the break-up time (BUT), and mean BUT was calculated by averaging values from both eyes. A result $<10 \mathrm{~s}$ was considered as abnormal. Intraocular pressure was measured using the Goldman applanation tonometer. A detailed slit-lamp examination of the anterior and posterior segments was performed. After pupil dilatation, the morphology of the optic disc was assessed by stereoscopic bio-microscopy. A pathologic optic disc was defined as optic nerve cupping and rim thinning (cup-disc ratio $\geqslant 0.6$ or cup-disc asymmetry $\geqslant 0.3) \pm$ partial or complete notching \pm rim haemorrhages.

\section{Topography and central corneal thickness}

All topography and central corneal thickness (CCT) measurements were performed using the Pentacam rotating Schiempflug camera, software version 1.15 (Oculus, Wetzlar, Germany). Topography criteria for Keratoconus diagnosis were central steepening, inferior or superior steepening, and skewing of the radial axis above or below the horizontal meridian. ${ }^{22}$ Diagnosis was confirmed by the presence of other disease features as defined by the Collaborative Longitudinal Evaluation of Keratoconus study, ${ }^{23}$ that is (1) an irregular cornea determined by distorted keratometry mires, distortion of the retinoscopic, or ophthalmological red reflex (or a combination of the two) and (2) at least one of the following biomicroscopic signs: Vogt's striae, Fleischer's ring of $>2 \mathrm{~mm}$ arc, and corneal scarring consistent with Keratoconus.

\section{Visual fields}

VF examination was carried out in all patients with suspicious disc changes and/or intraocular pressure 
$\geqslant 21 \mathrm{~mm} \mathrm{Hg}$. VF examination was performed with the Humphrey VF Analyzer equipped with Statpac 2 software (Zeiss Humphrey systems, San Leandro, CA, USA) and set for the central 24-2 threshold test.

Pathological VF were defined as an presence of one or more of the following abnormalities in the absence of other explanations: cluster of three adjacent depressed points on the pattern standard deviation plot at $P<5 \%$ level, a nasal step or scotoma, inferior or superior arcuate scotoma, paracentral scotoma, or generalised depression. Additional criteria were abnormal glaucoma hemifield test and/or a mean deviation $>5$.

Primary open angle glaucoma was diagnosed when the glaucomatous VF defect matched the optic disc changes with intraocular pressures $\geqslant 21 \mathrm{~mm} \mathrm{Hg}$ / (irrespective of the intraocular pressure) and normal, open anterior chamber angles. Ocular hypertension was defined as the presence of intraocular pressures of $>21 \mathrm{~mm} \mathrm{Hg}$ with normal disc, VFs, and open angles.

\section{Sleep studies}

All participants had been referred to the local sleep clinic (LD) and had undergone overnight oximetry. Limited sleep studies are performed (to differentiate obstructive and central events) in patients with significant cardiac history, signs of heart failure, and patients with neurological problems or taking significant doses of opioid medication. None of the 269 patients investigated in the study period required further assessment.

OSAHS was diagnosed in which the $4 \%$ oxygen desaturation rate was $>10$ events per hour. Disease severity was graded by the number of events per hour or the ODI. An ODI of $<5$ is considered as normal, 5-15 mild; $15-30$ moderate, and $>30$ events per hour characterises severe OSAHS.

\section{Statistical analysis}

Data was initially analyzed for differences among all patients with OSAHS and the controls (normal sleep study). Subgroup analysis was also undertaken for CPAP users and non-users. All continuous variables were described by mean (SD). Comparison of quantitative variables (e.g. age) was performed using an unpaired $t$-test. Comparison of proportions was made using $\chi^{2}$ test except in cases of small frequencies when Fisher's exact test was used. Spearman's rank correlations controlled for age and BMIs were determined between ODI and ocular symptoms, BUT, LDD, IOP, CCT, and astigmatism. A $P$-value of $<0.05$ was considered as statistically significant.

\section{Results}

The demographic, sleep, and ocular data of all OSAHS patients and controls are presented in Table 1 . The distribution of ocular irritation symptoms and BUT are presented in Figures 1 and 2, respectively. Eye rubbing, gritty sensation, mucoid discharge, and photophobia were significantly more frequent in the OSAHS patients $(P<0.05)$. Only four patients with OSAHS, but none of the controls, had experienced spontaneous lid eversion when sleeping, squeezing, or rubbing eyes. Two OSAHS patients had Keratoconus, both reported excessive eye rubbing and had increased lid laxity, but did not have frank FES. No patient or control was noted to have lacrimal gland prolapse, optic disc pallor, or swelling.

Seventy-five per cent $(67 / 89)$ of the OSAHS patients had been on CPAP for an average period of 19.6 (SD 15.3) months. The remaining 25\% (22/89) were intolerant or refused CPAP. The demographic, sleep, and ocular data of the two groups are compared in Table 2. Twenty-one per cent $(14 / 67)$ of CPAP users had experienced occasional mask edge leaks that resulted in ocular irritation and occasional episodes of conjunctivitis. A record of the change in ocular symptoms after use of CPAP showed improvement in 5/67 (7.4\%), worsening in 4/67 (5.9\%), but majority did not recall noticing any appreciable change $(58 / 67,86.6 \%)$ after initial adaptation or refitting of the mask. All patients and controls when queried on the sleep posture confirmed to be restless sleepers with variable sleep postures. However, all patients using CPAP since onset of therapy, slept on their backs to prevent mask displacements.

The dose-response relationships were also examined between ocular findings (such as IOP, CCT, etc.) and the severity of OSAHS using the Spearman's rank correlations. The ODI correlated significantly with number of ocular irritation symptoms, lid laxity, and average BUT, each correlation controlled for age and BMI (Table 3).

\section{Discussion}

Symptomatic OSAHS worthy of CPAP treatment has a prevalence of $1-2 \%$ in men, the prevalence in women being a third of this, both varying with the prevailing obesity. ${ }^{1}$ Several mechanisms have been implicated in the development of ocular changes and the effects of treatment with CPAP on the eye, the most common being related to the vascular and mechanical effects of the disease. ${ }^{3,14-19}$

Lid and ocular surface disorders are considered as secondary to the mechanical effects of eye rubbing and prone posture with contact of lid and palpebral conjunctiva to the pillow. The direct surface trauma 
Table 1 Demographic, sleep and, ophthalmic data of sleep apnoea patients and controls

\begin{tabular}{|c|c|c|c|}
\hline & Patients $(\mathrm{n}=89)$ & Controls $(\mathrm{n}=26)$ & *P-value \\
\hline Age (years) & $55.75 \pm 10.97$ & $55.3 \pm 10.7$ & 0.85 \\
\hline Median (range) & $56(21-82)$ & $55.5(33-75)$ & \\
\hline Gender $(\%)$ & 75 males $(84.3)$ & 17 males $(65.4)$ & 0.34 \\
\hline Body mass index $\left(\mathrm{kg} / \mathrm{m}^{2}\right)$ & $35.03 \pm 7.17$ & $32.9 \pm 5.15$ & 0.104 \\
\hline Median (range) & $34(25-58)$ & $32.5(25-43)$ & \\
\hline Oxygen desaturation index & $35.2 \pm 24.3$ & $3.08 \pm 2.8$ & $<0.001$ \\
\hline Median (range) & $30(10-65)$ & $2.5(0-9)$ & \\
\hline Ocular irritations symptoms (\%) & $71(79.8 \%)$ & $9(35 \%)$ & $<0.001$ \\
\hline Break-up time (s) & $8.52(6.8)$ & $11.15(4.6)$ & $<0.05$ \\
\hline Median (range) & $6.5(0-40)$ & $11(3-26)$ & \\
\hline Upper lid distraction (mm) & $8.4 \pm 3.1$ & $6.55 \pm 1.77$ & 0.001 \\
\hline Median (range) & $8(3-16)$ & $6(2.5-10)$ & \\
\hline Lower lid distraction $(\mathrm{mm})$ & $10.4 \pm 3.4$ & $8.28 \pm 2.08$ & $<0.001$ \\
\hline Median (range) & $10(5-20)$ & $8(4.5-13)$ & \\
\hline Floppy eyelid syndrome (\%) & $28(31.5)$ & $1(3.8)$ & 0.005 \\
\hline Intraocular pressure $(\mathrm{mm} \mathrm{Hg})$ & $15.4 \pm 2.9$ & $15.7 \pm 2.7$ & 0.629 \\
\hline Median (range) & $16(10-24.5)$ & $16(10-21.5)$ & \\
\hline Central corneal thickness $(\mu)$ & $539.2 \pm 53.5$ & $537 \pm 42.4$ & 0.842 \\
\hline Median (range) & $542(336-630)$ & $532.5(477-633.5)$ & \\
\hline Primary open angle glaucoma (\%) & $3(3.4)$ & $1(3.8)$ & 0.92 \\
\hline Ocular hypertension & $6(6.7)$ & $0(0)$ & 0.332 \\
\hline Astigmatism (diopters) & $1.12 \pm 0.88$ & $0.9 \pm 0.6$ & 0.272 \\
\hline Mean (range) & $0.95(0.13-5.2)$ & $0.7(0.25-3.2)$ & \\
\hline Keratoconus $(\%)$ & $2(2.2)$ & $0(0)$ & 1.000 \\
\hline
\end{tabular}

${ }^{*} P$-value: statistical significance of the difference between two groups.

The data is given as mean \pm standard deviation.

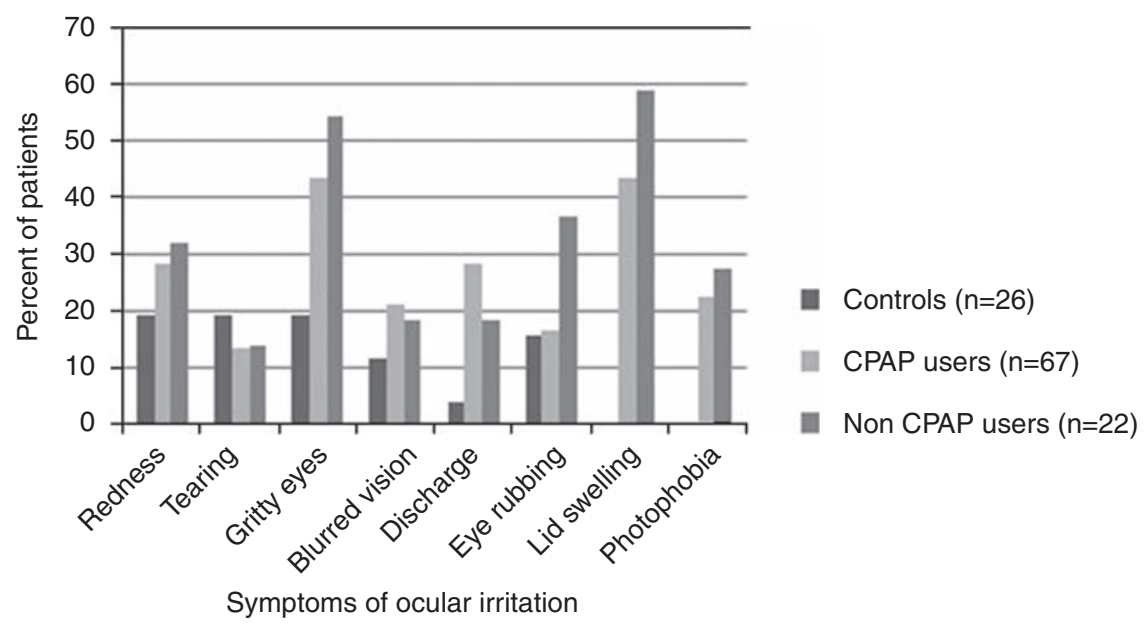

Figure 1 Ocular irritation symptoms in sleep apnoea patients (CPAP users and non-users) and controls.

induces chronic inflammation and tissue ischaemia with a possible up-regulation of elastolytic enzymes. ${ }^{24}$

Reduced tarsal elastin showed in FES by Netland et al ${ }^{25}$ supports the hypothesis, as does the presence of asymmetrical disease corresponding to the patients' sleep posture and resolution of symptoms with use of eye shield. ${ }^{5}$

The earliest association of OSAHS and FES was made by Gonnering and Sonneland in $1987 .{ }^{26}$ A decade later,
$\mathrm{McNab}^{5}$ reported his cumulative series of 50 FES patients out of which $48(96 \%)$ had history of sleep disturbed breathing and $26(96 \%)$ of the 27 patients undergoing polysomnography were confirmed to have OSAHS. Conversely, increased lid laxity has been associated with OSAHS and shown to have a positive correlation with disease severity. ${ }^{4,5,27,28}$ However, the reported prevalence of FES and ocular irritation symptoms in OSAHS has varied in different studies. The range of reported rates of 


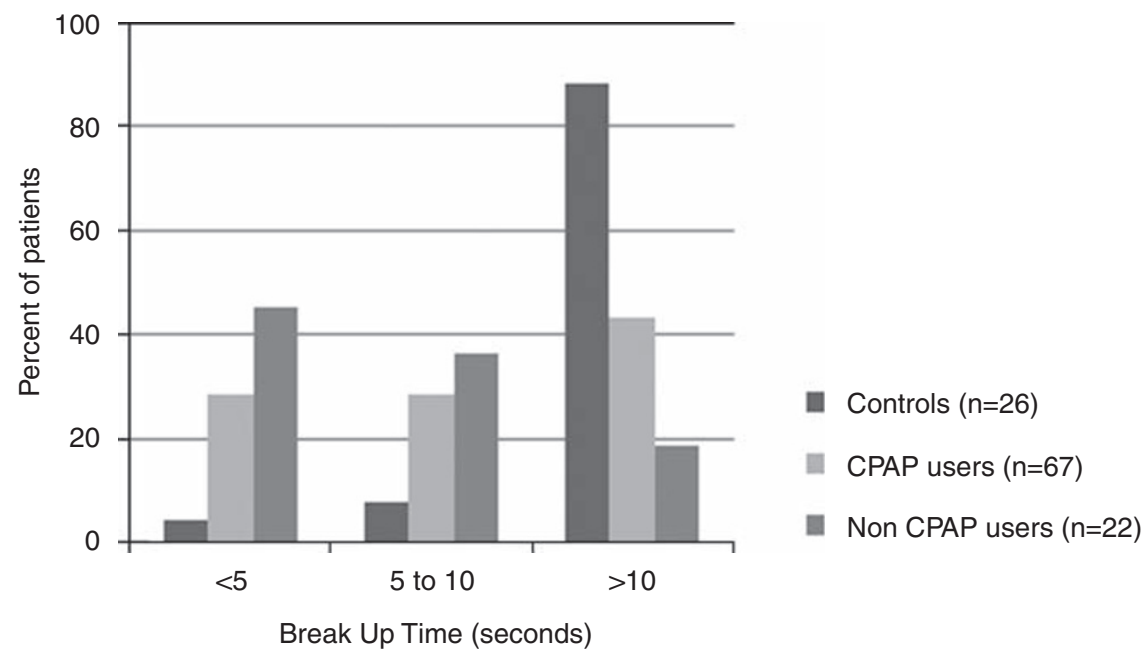

Figure 2 Distribution of tear BUT in sleep apnoea patients (CPAP users and non-users) and controls.

Table 2 Demographic, sleep, and ocular data of sleep apnoea patients: CPAP users and non-users

\begin{tabular}{|c|c|c|c|}
\hline & CPAP users $(\mathrm{n}=67)$ & No treatment $(\mathrm{n}=22)$ & *P-values \\
\hline Age (years) & $54.91 \pm 11.07$ & $58.32 \pm 10.48$ & 0.200 \\
\hline Median (range) & $56(21-82)$ & $59(37-79)$ & \\
\hline Gender: males (\%) & $58(86.56)$ & $16(72.7)$ & 0.135 \\
\hline Body mass index $\left(\mathrm{kg} / \mathrm{m}^{2}\right)$ & $34.5 \pm 6.59$ & $36.55 \pm 8.7$ & 0.330 \\
\hline Median (range) & $34(25-55)$ & $33.5(27-58)$ & \\
\hline Oxygen desaturation index & $35.06 \pm 24.38$ & $35.67 \pm 24.7$ & 0.920 \\
\hline Median (range) & $30(10-95)$ & $35(10-90)$ & \\
\hline Ocular irritation symptoms (\%) & $51(76)$ & $20(90.9)$ & 0.134 \\
\hline Break-up time (s) & $9.23 \pm 7.39$ & $6.38 \pm 4.2$ & 0.029 \\
\hline Median (range) & $8(0-40)$ & $5.5(2-18)$ & \\
\hline Upper lid distraction (mm) & $8.37 \pm 3.25$ & $8.59 \pm 2.5$ & 0.746 \\
\hline Median (range) & $8(3-16)$ & $8.5(5-13)$ & \\
\hline Lower lid distraction (mm) & $10.53 \pm 3.6$ & $10.2 \pm 2.77$ & 0.633 \\
\hline Median (range) & $10(5-20)$ & $10(8-15)$ & \\
\hline Floppy eyelid syndrome (\%) & $23(25.8)$ & $5(22.7)$ & 0.77 \\
\hline Intraocular pressure $(\mathrm{mm} \mathrm{Hg})$ & $15.55 \pm 2.9$ & $14.75 \pm 2.7$ & 0.239 \\
\hline Median (range) & $16(10-24.5)$ & $14(10-19.5)$ & \\
\hline Central corneal thickness $(\mu)$ & $550.8 \pm 45.7$ & $514 \pm 61.3$ & 0.018 \\
\hline Median (range) & $552(450-630)$ & $577(336-620.5)$ & \\
\hline Primary open angle glaucoma (\%) & $3(4.5)$ & $0(0)$ & 0.317 \\
\hline Ocular hypertension $(\%)$ & $6(8.9)$ & $0(0)$ & 0.151 \\
\hline Astigmatism (diopters) & $1.01 \pm 0.73$ & $1.44 \pm 1.17$ & 0.123 \\
\hline Median (range) & $0.85(0.13-4.2)$ & $1.1(0.3-2.35)$ & \\
\hline Keratoconus (\%) & $1^{\mathrm{a}}(1.5)$ & $1(4.5)$ & 0.412 \\
\hline
\end{tabular}

The data is given as mean \pm standard deviation.

${ }^{*} P$-value: statistical significance of the difference between two groups.

a Long-standing changes.

FES prevalence (2.3-53\%) in different series of OSAHS patients include Karger et al ${ }^{27} 1 / 44,2.3 \%$; Roberts et al, ${ }^{28}$ 1/46, 2.2\%; McNab, ${ }^{5} 3 / 20,15 \%$; Mojon et al, ${ }^{4} 14 / 44,32 \%$; and Sawatdiwithayayong, 53/100, 53\% (The association between severity of obstructive sleep apnoea and eyelid, conjunctiva and corneal abnormalities, poster presentation, Seri ARVO 2005.) All, but one of the studies (ie Robert $e a^{28}$ ), were conducted at the time of initial referral for OSAHS before initiation of CPAP. Robert et al in their series had a third of the OSAHS patients $(n=23)$ on CPAP.

To the best of our knowledge, ours is the largest cohort of OSAHS patients with moderate to severe disease $(n=89)$ and using CPAP $(75.3 \%, 67 / 89)$. OSAHS patients 
Table 3 Spearman correlations between oxygen desaturation index and ophthalmic findings

\begin{tabular}{lcc}
\hline & \multicolumn{2}{c}{ P-value $^{\mathrm{a}}$} \\
\cline { 2 - 3 } & $\begin{array}{c}\text { Sleep apnoea } \\
\text { patients, } \\
\mathrm{n}=89\end{array}$ & $\begin{array}{c}\text { Controls, } \\
\mathrm{n}=26\end{array}$ \\
\hline Ocular irritation symptoms & 0.05 & 0.868 \\
Upper lid distraction & 0.013 & 0.605 \\
Lower lid distraction & 0.031 & 0.721 \\
Average break-up time & 0.001 & 0.059 \\
Average intraocular pressure & 0.631 & 0.615 \\
Central corneal thickness & 0.769 & 0.487 \\
Average astigmatism & 0.083 & 0.079 \\
\hline
\end{tabular}

${ }^{\mathrm{a}}$ All correlations were controlled for age and body mass index.

in our study had significantly higher ocular irritation symptoms, abnormal BUT, lid laxity, and FES, as reported, earlier. However, CPAP users had lower ocular irritation and abnormal BUT compared with OSAHS patients not receiving treatment, although the difference was statistically significant only for BUT. There was no difference in the lid laxity between the two groups. Our findings may be explained by the CPAP users reporting the need to maintain supine posture at night, whereas those not on CPAP continued to have variable sleep postures. Mask edge leak did not appear to worsen symptoms in CPAP users. We propose that this effect was because long-term CPAP users had obtained better mask fits and become habituated to a supine sleep posture, thus reducing mask displacements and breaking the cycle of mechanical trauma and irritation.

Eye rubbing and mechanical trauma are also mechanisms implicated in development of Keratoconus. ${ }^{22,29}$ Both patients in our series with Keratoconus had increased lid laxity and were eye rubbers. One of them had not received any treatment, whereas the other one was on CPAP for 2 years, but the ocular changes were long standing.

Combinations of mechanical and vascular factors have a function in optic nerve pathology (glaucoma, anterior ischemic optic neuropathy, and disc swelling) reported with OSAHS. Vascular compromise can occur because of recurrent episodes of apnoea with reductions in arterial oxygen saturations, intermittent elevations in intracranial pressure, and systemic hypertension. ${ }^{1,2}$ Mechanical factors include an obesity-related increase in IOP. ${ }^{30}$ OSAHS is often, although not invariably, associated with increased BMI. The positive association between obesity and intraocular pressure is proposed to be related to increased blood viscosity, increased episcleral venous pressure, and decreased aqueous outflow. ${ }^{30}$
We did not have any patient in our cohort groups with disc swelling or pallor indicative of earlier episodes of ischemic optic neuropathy (NAION). Strong association of OSAHS with NAION has been shown in series of NAION patients when surveyed for symptoms of sleep disturbed breathing and/or on investigation with polysomnography. ${ }^{31-33}$ The role of OSAHS in the NAION disease mechanism appears significant and at the least a sleep history should be included in the work-up of patients with optic neuropathy.

Weaker evidence exists for prevalence of NAION in OSAHS patients. Mojon et $a l^{9}$ investigated a small series of seven OSAHS patients and four among them had nasal arcuate defects, two with additional paracentral relative defects. All four patients with abnormal fields had moderate to severe disease. The authors considered the VF changes to be consistent with optic neuropathy. They also observed the VFs changes to be stable over 18 months after initiation of CPAP, and concluded that therapy for OSAHS could reduce risk of optic neuropathy. Behbehani et al, ${ }^{34}$ however, questioned the adequacy of CPAP in preventing NAION. They reported NAION occurring in three patients when on treatment with CPAP for established OSAHS. In our study, although the patients included had moderate to severe disease, majority were on treatment. This may have reduced their risk of development of NAION. Our results also do not confirm reports of CPAP causing NAION. ${ }^{34}$

Similarly, the prevalence of glaucoma in our study was low $(3 / 89,3.4 \%)$ and no higher than the expected prevalence in the population (1.7-3\%), $P=0.429 .{ }^{35}$ Our observations were similar to those made by Geyer $e^{2} a^{36}$ and Pearson. ${ }^{37}$ The low prevalence in ours and Geyer et $a l^{\prime}$ 's studies could be the effect of CPAP treatment. Whereas, most other studies reporting higher prevalence of glaucoma in OSAHS (7.2-27\%) $)^{3,6-8,28,38}$ have investigated patients at time of referral for polysomnography and before initiation of therapy; Geyer recruited patients earlier diagnosed with OSAHS and possibly receiving treatment. The authors, however, have not mentioned the treatment status of the OSAHS patients in their report. ${ }^{36}$ Further reasons for a variable prevalence being reported can be the definitions used for glaucoma and study populations. We did not find our diagnostic criteria to vary significantly to earlier reports, and we consider our cohort of patients with significant OSAHS to be a relevant group for reporting prevalence of any associated pathology attributed to the sleep disorder.

A recent study reported significant 24 -h fluctuations in IOP, with the highest values at night. ${ }^{17} \mathrm{CPAP}$ therapy caused an additional IOP increase, especially at night. The authors recommend a regular screening 
of VFs and optic disc in all OSAHS patients, especially those treated with CPAP. This theoretical increase in risk of pathological disc changes with long-term CPAP was not corroborated by our results. However, we did not undertake routine VFs testing for all our patients.

Limitations of our study can include a referral bias in the population studied, as patients recruited were those referred for investigation for sleep disorder and may not be representative of the entire OSAHS population, majority of whom remain undiagnosed. The referred group of patients would include those with significant symptoms and identified as suspects by the general practitioner in the community. But as our results reflect the ocular findings in patients with significant disease (all patients included had ODI $>10$ ), they are likely to be more clinically relevant. Similarly, pulse oximetry was used routinely for screening OSAHS suspects in our sleep clinic. Limited sleep studies to assess respiratory events are considered as adequate first line method of diagnostic assessment for OSAHS. ${ }^{39}$ Oximetry alone has been shown to have a mean sensitivity of $87 \%$ and mean specificity of $65 \%$ when compared with the gold standard full polysomnography. ${ }^{40}$ This data suggests that pulse oximetry can be a useful investigation to positively diagnose OSAHS, although may have limitations in disease exclusion in patients with milder symptoms. ${ }^{39}$ Our cohort of confirmed OSAHS patients among the referrals was, therefore, likely to be under-diagnosed, but include the more clinically relevant subgroup of patients with significant disease.

In addition, the effects of CPAP showed by our results were not longitudinally assessed and may contain a population bias. However, with the current evidence and guidelines on management of OSAHS with CPAP, setting up of a randomised clinical trial to show these benefits in a longitudinal study would not be ethically appropriate. Further studies are also needed to clarify the long-term outcomes of CPAP initiated in patients with pre-existing ocular disease.

In conclusion, we consider our findings to be of importance as they suggest a benefit of treatment of OSAHS with CPAP on the ocular manifestations. The increased prevalence of ocular symptoms and signs in patients with OSAHS indicates a need to increase awareness and establish close collaboration with the sleep physicians with clear pathways for review of OSAHS patients by the ophthalmic services.

\section{Conflict of interest}

The authors declare no conflict of interest.

\section{References}

1 Stradling JR, Davies RJO. Sleep 1: obstructive sleep apnoea / hypopnoea syndrome: definitions, epidemiology, and natural history. Thorax 2004; 59: 73-78.

2 Schlosshan D, Elliott MW. Sleep 3: clinical presentation and diagnosis of the obstructive sleep apnoea hypopnoea syndrome. Thorax 2004; 59: 347-352.

3 McNab AA. The eye and sleep. Clin Experiment Ophthalmol 2005; 33: 117-125.

4 Mojon DS, Goldblum D, Fleischhauer J, Chiou AGY, Freuh BE, Hess CW et al. Eyelid, conjunctival and corneal findings in sleep apnoea syndrome. Ophthalmology 1999; 106: $1182-1185$.

5 McNab AA. Floppy eyelid syndrome and obstructive sleep apnea syndrome. Ophthalmic Plast Reconstr Surg 1997; 13: 98-114.

6 Mojon DS, Hess CW, Goldblum D, Fleischhauer J, Koerner F, Bassetti C et al. High prevalence of glaucoma in patients with sleep apnoea syndrome. Ophthalmology 1999; 106: 1009-1012.

7 Mojon DS, Hess CW, Golblum D, Bohnke M, Korner F, Mathis J. Primary open-angle glaucoma is associated with sleep apnoea syndrome. Ophthalmologica 2000; 214: 115-118.

8 Mojon DS, Hess CW, Goldblum D, Boehnke M, Koerner F. Normal-tension glaucoma is associated with sleep apnoea syndrome. Ophthalmologica 2002; 216: 180-184.

9 Mojon DS, Mathis J, Zulauf M, Koerner F, Hess CW. Optic neuropathy associated with sleep apnea syndrome. Ophthalmology 1998; 105: 874-877.

10 Tsang CSL, Chong SL, Ho CK, Li MF. Moderate to severe obstructive sleep apnoea patients is associated with a higher incidence of visual field defect. Eye 2006; 20: 38-42.

11 Purvin VA, Kawasaki A, Yee RD. Papilledema and obstructive sleep apnea syndrome. Arch Ophthalmol 2000; 118: 1626-1630.

12 Bucci FA, Krohel GB. Optic nerve swelling secondary to the obstructive sleep apnoea syndrome. Am J Ophthalmol 1986; 105: 428-430.

13 Sullivan CE, Isa FW, Besthon-Jones M, Eves L. Reversal of obstructive sleep apnoea by continuous positive airway pressure applied through the nares. Lancet 1981; 4: 862-865.

14 Parunovic A. Floppy eyelid syndrome. Br J Ophthalmol 1983; 67: 264-266.

15 McNab AA. Reversal of floppy lid syndrome with treatment of obstructive sleep apnoea. Clin Experiment Ophthalmol 2000; 28(2): 125-126.

16 Sebastian RT, Johns S, Gibson RA. Treating obstructive sleep apnoea syndrome: does it improve visual field changes? Eye 2006; 20: 118-120.

17 Kiekens S, De Groot V, Coeckelbergh T, Tassignon MJ, van de Heyning P, De Backer W et al. Continuous positive airway pressure therapy is associated with an increase in intraocular pressure in obstructive sleep apnoea. Invest Ophthal Vis Sci 2008; 49: 934-940.

18 Stauffer JL, Fayter NA, MacLurg BJ. Conjunctivitis from nasal CPAP apparatus (letter). Chest 1984; 86: 802.

19 Fayers T, Simcock DE, Wilkins MR. Reactivation of recurrent corneal erosion syndrome by continuous positive pressure ventilation. Cornea 2007; 26: 1292.

20 Liu D, Stasior OG. Lower eyelid laxity and ocular symptoms. Am J Ophthalmol 1983; 95: 545-551.

21 Culbertson WW, Ostler HB. The floppy eyelid syndrome. Am J Ophthal 1981; 92: 568-575. 
22 Rabinowitz YS. Keratoconus: update and new advances. Surv Ophthalmol 1998; 42: 297-319.

23 Zadnik K, Barr JY, Edrington TB, Everett DF, Jameson M, McMahon TTet al. Collaborative Longitudinal Evaluation of Keratoconus (CLEK) Study Group. Baseline findings in the Collaborative Longitudinal Evaluation of Keratoconus (CLEK) study. Invest Ophthal Vis Sci 1998; 39: 2537-2546.

24 Schlotzer-Schrehardt U, Stojkovic M, Hofmann-Rummelt C, Kruse FE, Holbach LM. The pathogenesis of floppy eyelid syndrome: involvement of matrix metalloproteinases in elastic fiber degradation. Ophthalmology 2005; 112: 694-704.

25 Netland PA, Sugrue SP, Albert DM, Shore JW.

Histopathologic features of the floppy eyelid syndrome. Ophthalmology 1994; 101: 174-181.

26 Gonnering RS, Sonneland PR. Meibomian gland dysfunction in floppy eyelid syndrome. Opthal Plast Reconstr Surg 1987; 3: 99-103.

27 Karger RA, White WA, Park WC, Rosakles AG, McLaren JW, Olson EJ et al. Prevalence of floppy eyelid syndrome in obstructive sleep apnoea hypopnoea syndrome. Ophthalmology 2006; 113: 1669-1674.

28 Robert PY, Adenis JP, Tapie P, Melloni B. Eyelid hyperlaxity and obstructive sleep apnea (OSA) syndrome. Eur J Ophthalmol 1997; 7: 211-215.

29 Donnenfield ED, Perry HD, Gibralter RP, Ingraham HJ, Udell IJ. Keratoconus associated with floppy eyelid syndrome. Ophthalmology 1991; 98: 1674-1678.

30 Cheung N, Wong TY. Obesity and eye diseases. Surv Ophthalmol 2007; 52: 180-195.

31 Mojon DS, Hedges TR, Ehrenberg B, Karam EZ, Goldblum D, Abou-Chebl A et al. Association between sleep apnoea syndrome and nonarteritic anterior ischemic optic neuropathy. Arch Ophthalmol 2002; 120: 601-605.

32 Palombi K, Renard E, Levy P, Chiquet C, Deschaux Ch, Romanet JP et al. Non-arteritic anterior ischaemic optic neuropathy is nearly systemically associated with obstructive sleep apnoea. Br J Ophthalmol 2006; 90: 879-882.

33 Li J, McGwin G, Vaphiades MS, Owsley C. Non-arteritic anterior ischaemic optic neuropathy and presumed sleep apnoea syndrome screened by the sleep apnoea scale of sleep disorders questionnaire (SA-SDQ). Br J Ophthalmol 2007; 91: 1524-1527.

34 Behbehani R, Mathews MK, Sergott RC, Savino PJ. Non arteritic anterior ischaemic optic neuropathy in patients with sleep apnoea while being treated with continuous positive airway pressure. Am J Ophthalmol 2005; 139: 518-521.

35 Quigley HA. Number of people with glaucoma worldwide. Br J Ophthalmol 1996; 80: 389-393.

36 Geyer O, Cohen N, Segev E, Rath EZ, Melamud L, Peled R et al. The prevalence of glaucoma in patients with sleep apnea syndrome: same as in the general population. Am J Ophthalmol 2003; 136: 1093-1096.

37 Pearson J. Glaucoma in patients with sleep apnoea. Ophthalmology 2007; 5: 816.

38 Bedel RE, Kaplan J, Heckman M, Friedrickson PA, Lin S-C. Prevalence of glaucoma in patients with obstructive sleep apnoea - a cross-sectional case-series. Eye 2008; 22: 1105-1109.

39 Management of Obstructive Sleep Apnoea/Hypopnoea Syndrome in Adults. Scottish Intercollegiate Guidelines Network (June 2003).http://www.sign.ac.uk. Accessed 24.06.2009.

40 Ross SD, Allen IE, Harrison KJ, Kvasz M, Connelly J, Sheinhart IA. Systematic review of literature regarding the diagnosis of sleep apnoea. Rockville (MD). Agency for Health Care Policy and Research 1999. AHRQ Evidence report 1. http://www.ncbi.nlm.nih.gov/books/ bv.fcgi?rid=hstat1.chapter.2. Accessed 24.06.2009. 